\title{
Structures of Butylthiolate Self-Assembled Monolayers on Au(111) with Gold Adatoms
}

\author{
Seol Ryu, Jeewon Kang, ${ }^{\dagger}$ Young-Kyu Han, ${ }^{\ddagger, *}$ and Yoon Sup Lee ${ }^{\dagger, *}$ \\ Department of Chemistry, Chosun University, Gwangju 501-759, Korea \\ †Department of Chemistry, KAIST, Daejeon 305-701, Korea. E-mail: yslee@kaist.edu \\ "Division of Materials Science, Korea Basic Science Institute (KBSI), Daejeon 305-333, Korea.*E-mail: ykhan@kbsi.re.kr \\ Received July 11, 2011, Accepted August 8, 2011
}

\begin{abstract}
A density functional theory method with the local basis set was employed to perform slab calculations to study thiolate-induced surface reconstruction structures of butylthiolates (ButS) with $c(4 \times 2)$ superlattice of the $\mathrm{Au}(111)$ surface. The slab calculations indicate that the most stable adsorption structure is the ButS-Au (adatom)-SBut complex form, which is in good agreement with the reported experiments and theoretical results for thiolates with shorter alkyl chains. The cis form of ButS-Au (adatom)-SBut motifs is preferred by $0.11 \mathrm{eV}$ with respect to the trans form, and by $0.15 \mathrm{eV}$ over the mixed cis-trans configurations due to the steric hindrance between adjacent butyl groups. It appears that the motif of Au adatom on the $\mathrm{Au}(111)$ surface is favored even for butylthiolate.
\end{abstract}

Key Words : Butylthiolate self-assembled monolayer, Surface slab calculation, Local basis set, Au adatom on Au surface

\section{Introduction}

Alkylthiolate (RS) self-assembled monolayers (SAMs) on the $\mathrm{Au}(111)$ surface have attracted wide interest over the past decades because of their great number of potential applications in nanotechnology, especially in molecular electronics, biochemical sensors, corrosion prevention, friction control, biocompatible materials, and so on. ${ }^{1-4}$ The process of RS molecule self-assembly on metal surfaces is governed by both the intermolecular interactions and the anchoring bond to the surface. The interaction between the RS chains is a weak van der Waals (vdW) type, whereas the anchoring is a chemical bond with a certain degree of ionic character. The closely packed alkylthiolates SAMs on $\mathrm{Au}(111)$ are ordered in a hexagonal $(\sqrt{3} \times \sqrt{3}) R 30^{\circ}$ lattice structure and this is confirmed by a variety of experimental techniques. ${ }^{5-8}$ Subsequently performed helium diffraction ${ }^{9}$ and grazing incidence X-ray diffraction (GIXRD) ${ }^{10}$ experiments revealed the existence of another stable and ordered surface structure, $c(4 \times 2)$ superlattice of the $(\sqrt{3} \times \sqrt{3}) R 30^{\circ}$ (whose reduced unit cell is a $(\sqrt{3} \times 2 \sqrt{ } 3)$ rect., containing four thiolate molecules in unit mesh). Both the $(\sqrt{3} \times \sqrt{3}) R 30^{\circ}$ and the $(\sqrt{3} \times 2 \sqrt{3})$ rect. phases generally coexist in spatiallydistinct domains and facile transformation between two phases has been observed. ${ }^{11,12}$

The long-standing question of the RS adsorption site has been rigorously studied over the past two decades. Most of the theoretical works have favored the occupation of the RS head group atom on either a bridge ${ }^{13,14}$ or an intermediate hollow-bridge ${ }^{15-18}$ of a flat gold surface. Until then, it was generally accepted that adsorption of alkylthiolate (RS) lifts the herringbone surface reconstruction of a clean $\mathrm{Au}(111)$ surface. The experimental works using photoelectron diffrac- tion $(\mathrm{PD})^{19}$ and normal incidence X-ray standing wave $(\text { NIXSW })^{20}$ discovered that RS chains adsorbed on the atop site. This atop has been the most unstable adsorption site for the previously performed density functional theory (DFT) calculations. ${ }^{15,16,18}$ Lately, these large discrepancies between experiments and theoretical works can be explained by introducing a new concept of thiolate-induced surface reconstruction. ${ }^{11}$ As a result, based on the NIXSW ${ }^{21}$ and STM $^{22}$ measurements, the new scenario of atop adsorption suggests that the $\mathrm{S}$ atom occupies a local atop site relative to a surface $\mathrm{Au}$ atom and that $\mathrm{RS}$ is bonded to $\mathrm{Au}$ adatoms, which have been lifted up from the gold substrate.

Theoretical calculations have also been performed with several new models of $c(4 \times 2)$ superstructure, which comprise the thiolate-adatom species, such as Au adatom-thiolate (RSAu), ${ }^{12,21-24}$ honeycomb (HC), ${ }^{25}$ (RSAu) $x$ polymer, ${ }^{26} \mathrm{Au}$ adatom-vacancy, ${ }^{27,28}$ and $\mathrm{Au}$ adatom-dithiolate (RS-Au$\mathrm{SR}),{ }^{22,27}$ in order to clarify and go with the atop adsorption scenario. Among them, RS-Au-SR complexes have been predicted as the most energetically favored model by Grönbeck et al. ${ }^{29}$

In this work, we chose medium-length alkylthiolates, $\mathrm{CH}_{3}\left(\mathrm{CH}_{2}\right)_{3} \mathrm{~S}$ (ButS, butylthiolates), for adsorbate species on the $\mathrm{Au}(111)$ surface and calculated their adsorption structures and stabilities on the $c(4 \times 2)$ superlattice of the surface with the adatom system. Various adatom-containing reconstructed models, as well as a flat surface model, were considered to find the most energetically favorable reconstruction model. Use of the medium length of ButS may lead to more realistic adsorption structures in which possible chain length effects are reflected, while most of the previous theoretical studies were done with rather short thiols.

Computational Details. All the calculations were per- 
formed using the SIESTA code $\mathrm{e}^{30,31}$ which implements the DFT method with the linear combination of atomic orbitals (LCAO) approximation. The exchange-correlation energy was expressed with the generalized gradient approximation $(\mathrm{GGA}){ }^{32-35}$ using the Perdew-Burke-Ernzerhof $(\mathrm{PBE})^{36}$ functional. Core electrons are represented by norm-conserving pseudopotentials, using the Troullier-Martins scheme $\mathrm{e}^{37}$ with relativistic correction. For valence electrons, we have used a split-valence double- $\zeta$ basis set, including polarization (DZP) orbitals for all atoms, as obtained with an energy shift of $20 \mathrm{meV}$. The calculated bulk lattice parameter for $\mathrm{Au}$ is $4.22 \AA$, which is slightly larger than the experimental values of $4.08 \AA$, and in good agreement with the previous DFT calculations. ${ }^{16,17,26,38}$

The $c(4 \times 2)$ superlattice was used to describe the $\mathrm{Au}(111)$ surface and it was modeled as a repeated slab geometry consisting of six layers of $\mathrm{Au}$ atoms and a vacuum region of about $12 \AA$ from the top of the thiolate to the bottom of the $\mathrm{Au}$ layer of the next slab. Four ButS were adsorbed on one side of the slab, corresponding to the coverage of $0.33 \mathrm{~mL}$. The maximum kinetic energy of the plane wave is $200 \mathrm{Ry}$. The Brillouin zone integration was performed with a $(5 \times 5$ $\times 1)$ Monkhorst-Pack ${ }^{39}$ k-grid mesh. Electronic energies were calculated with the SCF tolerance of $10^{-4} \mathrm{eV}$. The geometry of the structure was optimized until residual forces became less than $0.03 \mathrm{eV} / \AA$. The optimizations were performed by fully relaxing the coordinates of the alkylthiolates and the Au atoms, except for the bottom three layers of the slab. The basis set superposition error (BSSE) correction for adsorption energy was not considered because the main focus of this work is on the relative stabilities of various adsorption structures, each of which is subject to about the same degree of BSSE.

\section{Results and Discussion}

We have investigated nine adsorption structures as reconstructed $\mathrm{Au}(111)$ surface models (see Figure 1), and summarized the structural and energetic properties in Table 1.

Table 1. The adsorption energy, the average distance of Aux-S (X: adatom and surface Au atoms), S-C, and the angle of the S-C bond with respect to the surface normal of ButS adsorbed on $\mathrm{Au}(111)$

\begin{tabular}{lccccc}
\hline & $\begin{array}{c}\mathrm{E}_{a d} \\
(\mathrm{eV})\end{array}$ & $\begin{array}{c}d_{\text {Auad-S }} \\
(\AA)^{a}\end{array}$ & $\begin{array}{c}d_{\text {Ausurf-S }} \\
(\AA)^{b}\end{array}$ & $\begin{array}{c}d_{\text {S-C }} \\
(\AA)\end{array}$ & $\begin{array}{c}\theta_{\text {S-C }} \\
\left({ }^{\circ}\right)\end{array}$ \\
\hline (a) fcc-brd & 1.77 & & 2.542 & 1.864 & 57.1 \\
(b) fcc & 1.32 & 2.321 & & 1.844 & 65.0 \\
(c) HC & 1.79 & & 2.474 & 1.862 & 46.5 \\
(d) pol & 1.72 & 2.485 & & 1.863 & 51.6 \\
(e) 1ad+1va & 1.80 & 2.365 & $2.514 / 2.557$ & 1.854 & 55.4 \\
(f) pol+2va & 1.52 & 2.432 & 2.504 & 1.848 & 57.4 \\
(g) $(\mathrm{RS})_{2}$-cis & 1.93 & 2.373 & 2.572 & 1.856 & 53.3 \\
(h) $(\mathrm{RS})_{2}$-trans & 1.82 & 2.358 & 2.609 & 1.859 & 55.5 \\
(i) (RS) $)_{2}$-mix & 1.78 & 2.369 & 2.594 & 1.861 & 50.2 \\
\hline
\end{tabular}

${ }^{a}$ Distance between $\mathrm{S}$ and an $\mathrm{Au}$ adatom. ${ }^{b}$ Distance between $\mathrm{S}$ and a substrate $\mathrm{Au}$ atom.
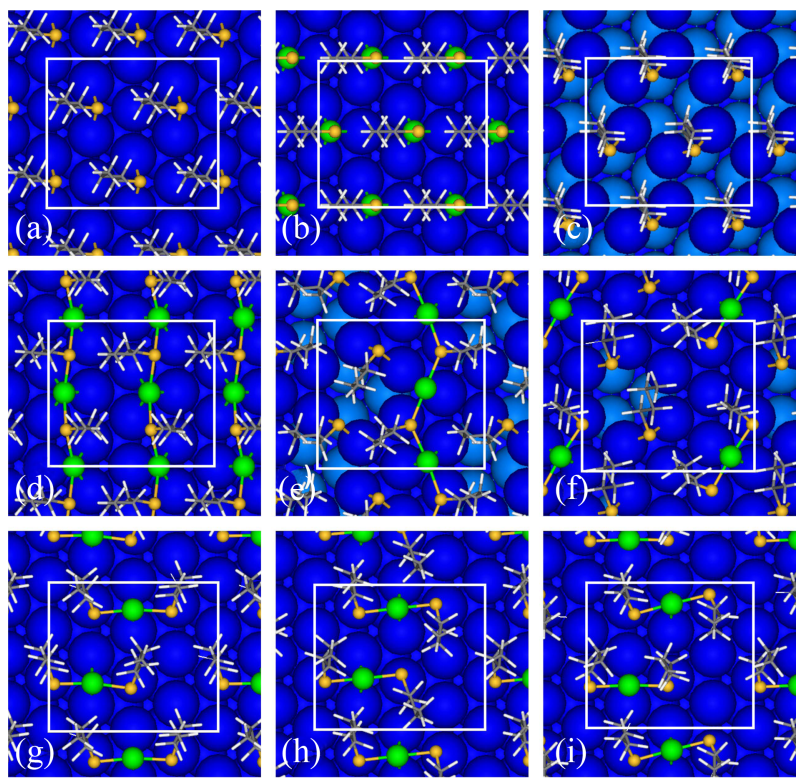

Figure 1. Top view of the optimized structure of ButS on $\mathrm{Au}(111)$. The surface $c(4 \times 2)$ unit cell is shown with a white line. Color code: blue $(\mathrm{Au})$, green $(\mathrm{Au}$ adatoms), yellow $(\mathrm{S})$, gray $(\mathrm{C})$, and white $(\mathrm{H})$.

Figure 1(a) represents the adsorption structure of four butylthiolate (ButS) adsorbed on an unreconstructed $\mathrm{Au}(111)$ surface of fcc-bridge (fcc-brd) sites, which are the most stable adsorption structure of alkylthiolate in a $(\sqrt{3} \times$ $\sqrt{ } 3) R 30^{\circ}$ periodic lattice. ${ }^{15-18}$ For the atop-adatom $c(4 \times 2)$ models, (b)-(i), we calculated adsorption structures that have been suggested by the experimental and theoretical works. ${ }^{12,21-29}$ As an adatom-thiolate (RSAu) model, ${ }^{12,21,23,24}$ (b), where RSAu is on top of an Au adatom, we have adsorbed $\mathrm{Au}$ adatoms occupying threefold coordinated hollow sites, fcc, which was suggested by the NIXSW experiments. ${ }^{21}$ Panel (c) is a honeycomb (HC) model, ${ }^{25}$ which has one defect for every three $\mathrm{Au}$ atoms. The ad-pol, (d), represents the $(\mathrm{RSAu})_{x}$ polymer model in which one $\mathrm{Au}$ atom is bonded to the surface in a fcc position, and the other occupies a hcp position while thiolate species bridge these adatoms. ${ }^{26}$ For models that contain adatoms or $(\mathrm{RSAu})_{x}$ polymers with surface vacancies, (e)-(f), 1ad+1va and pol+2va are investigated. ${ }^{27,28}$ The packing structure shown in Figure 1(e), the 1ad+1va model, demonstrates that two of the four thiolates are bound to the Au adatom and occupy an atop site relative to the underlying $\mathrm{Au}(111)$ surface, while the other two are at bridge sites. The panel ( $\mathrm{f}$ ) has a polymeric zigzag chain and two vacancies in the unit cell. ${ }^{27}$ For (g)-(i), Au adatom-dithiolate (RS-Au-SR) complexes, ${ }^{22,27,29}$ where $\mathrm{Au}$ adatom occupies a bridge site on the $\mathrm{Au}(111)$ surface and two thiolates are bonded to opposite sides of this adatom, such that they occupy the nearest atop sites relative to the underlying Au surface atoms, are calculated. Depending on the relative orientation of the two butyl groups, two complexes were divided into cis and trans configurations, and we have named these (RS) 2 -cis and (RS) $)_{2}$-trans, respectively. 
The relative stability was evaluated by comparing an adsorption energy between the adopted model and the standard adsorption structure of (a), four ButS on the fcc-brd site. The adsorption energy $\left(\mathrm{E}_{a d}\right)$ is calculated as:

$$
\begin{aligned}
\mathrm{E}_{a d}=\frac{1}{n_{B u t S}} & ([\mathrm{E}(\mathrm{ButS} / \mathrm{Au}(111)]-\mathrm{E}[\mathrm{Au}(111)]-\mathrm{E}[\mathrm{ButS}] \\
& \left.\times \mathrm{n}_{B u t S}-\mathrm{E}[\mathrm{Au}(\text { bulk })] \times \mathrm{n}_{a d}\right)
\end{aligned}
$$

where $\mathrm{E}[\mathrm{ButS} / \mathrm{Au}(111)], \mathrm{E}[\mathrm{Au}(111)]$, and $\mathrm{E}[\mathrm{ButS}]$ are the total energy of ButS adsorbed on $\mathrm{Au}(111)$, the isolated $\mathrm{Au}(111)$ slab, and the isolated ButS molecule, respectively. The $\mathrm{n}_{\text {But }}$ and $\mathrm{n}_{\text {ad }}$ values are the number of ButS molecules and the number of $\mathrm{Au}$ adatoms in a unit cell, respectively. These follow the concept of Grönbeck et al., ${ }^{29}$ which regards bulk $\mathrm{Au}$ as a metal atom reservoir.

The adsorption energy of ButS on top of the adatom, (b) fcc, is energetically less stable by $0.44 \mathrm{eV}$ than that of the unreconstructed surface model, (a) fcc-brd. This result is in line with the discussion of the energetic penalty of the creation of adatoms for the adatom-thiolate (RSAu) model. ${ }^{29}$ These smaller adsorption energies support the inadequacy of $\mathrm{Au}$ adatom-thiolate (RSAu) models, (b), as the proper reconstructed adsorption structure which can support the experimental results. Furthermore, (f), pol+2va is also less favored by $0.25 \mathrm{eV}$. Because of the strained configuration, the binding energy does not compensate for the energetic penalty connected to the formation of the point defects in (f). ${ }^{40}$ In contrast to these models, the honeycomb (HC), (c), $(\mathrm{RSAu})_{x}$ polymer $(\mathrm{pol}),(\mathrm{d})$, adatom-vacancy $(1 \mathrm{ad}+1 \mathrm{va}),(\mathrm{e})$, and $\mathrm{Au}$ adatom-dithiolate models are favored over the unreconstructed surface model, (a). Au adatom-dithiolate (RS-Au-SR), with the cis configuration, $(\mathrm{RS})_{2}-c i s,(\mathrm{~g})$, is the

(a)

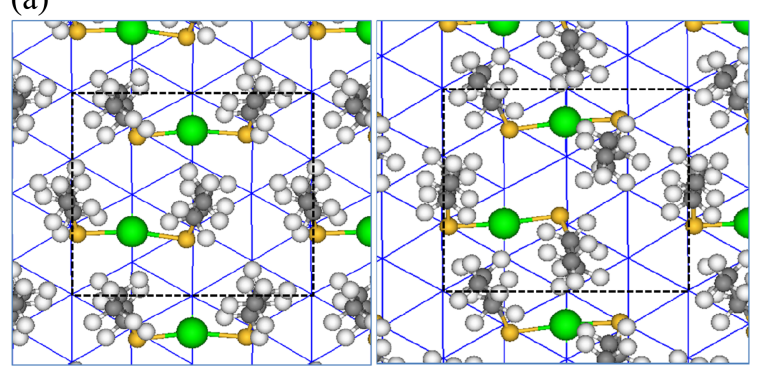

(b)

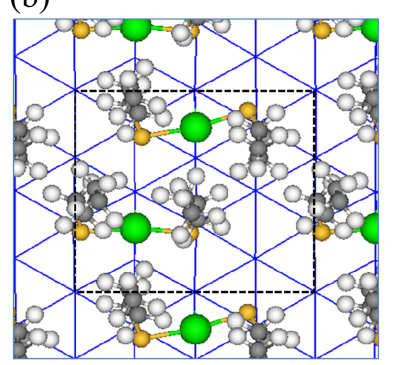

Figure 2. Top view of the optimized structure of ButS-Au-SBut on $\mathrm{Au}(111)$. The surface $(2 \sqrt{3} \times 3)$ unit cell is shown with a dotted line, (a) Left: (RS) $)_{2}$-cis, right: (RS) $)_{2}$-trans model, and (b) (RS) $)_{2}$-mix model. most preferred adsorption structure among the considered nine models with $\mathrm{E}_{a d}=1.93 \mathrm{eV}$. Between two adsorption configurations, the preference of the $c i$ form is explained by the steric hindrance caused by the close proximity of the two butyl groups in the trans form. ${ }^{41}$ In Figure 2(a), we have directly compared the packing configurations of cis and trans ButS-Au-SBut. It is seen that alkyl chains, with a cis configuration, are highly ordered and packed better than any of those with the trans configuration. The shortest distance between butyl groups in the cis and trans form is 2.1-2.4 $\AA$ and 1.8-2.1 $\AA$, respectively. In order to reduce the steric repulsion and obtain a more regular form of the packing structure, we expect that the RS-Au-SR with cis configuration is highly preferred over the trans configuration. The $\mathrm{RS}-\mathrm{Au}-\mathrm{SR}$ group, the so-called staple motif, is also energetically favored on thiolate-protected gold nanoparticles. ${ }^{42}$ Moreover, this motif is expected to be highly mobile on $\mathrm{Au}(111)$ and undergoes facile change between two cis and trans configurations. ${ }^{33,36,38}$ Therefore, we have calculated the intermediate state for the first time, using mixed cis and trans configurations and (RS) ${ }_{2}$-mix (see Figure 2(b)). The steric repulsion is also present in the mixed form since the shortest distance between butyl groups is 1.8-2.2 $\AA$ and the mixed form is $0.15 \mathrm{eV}$ higher in energy than the stable cis configuration.

The structural features are summarized in Table 1. Experimentally observed $\mathrm{R}(\mathrm{S}-\mathrm{Au})$ is $2.5 \AA$ with the $\mathrm{S}-\mathrm{C}$ bond axis tilted by $50^{\circ} \cdot{ }^{19,20}$ For the (RSAu) model, (b), the average bond length between the $\mathrm{Au}$ adatom and the $\mathrm{S}$ of thiolate,

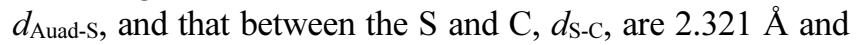
$1.844 \AA$, respectively. The optimized tilting angle of ButS $65.0^{\circ}$, is somewhat larger than that of the experimental adsorption structure. The bond length of the Au adatom and the ButS, $d_{\text {Auad-s, }}$, of the polymer model, $2.485 \AA$, is longer than any other calculated models, but quite similar to the value of the unreconstructed surface model, (a), $2.542 \AA$. For $1 \mathrm{ad}+1 \mathrm{va},(\mathrm{e})$, the distance between the Au adatom and the sulfur atom, $d_{\text {Auad-s, }}$, is $2.365 \AA$, and the average distance between the surface $\mathrm{Au}$ atom and the sulfur atom, $d_{\text {Ausurf-S }}=$ $2.536 \AA$. The average distances of the two RS-Au-SR complexes have $d_{\text {Auad-S }}=2.373 \AA$ and $d_{\text {Ausurf-s }}=2.572 \AA$, which are slightly longer than experimentally observed values at the low coverage surface, $2.33 \AA$ and $2.49 \AA$, respectively. ${ }^{22}$ The average angle of $\angle \mathrm{S}-\mathrm{Au}-\mathrm{S}$ is $167.9^{\circ}$ and $166.7^{\circ}$, while the $\angle \mathrm{Au}-\mathrm{S}-\mathrm{Au}$ is $92.7^{\circ}$ and $89.9^{\circ}$ for $\mathrm{cis}$ and trans configuration, respectively. ${ }^{43}$ Unlike the Au adatomrelated distance terms, the $d_{\text {Aux-S }}, d_{\text {S-C }}$ bond is almost same for all investigated models. However, the S-C tilting angle, $\theta_{\mathrm{S}-\mathrm{C}}$, is highly dependent upon the adsorption structure and energy. For example, the Au adatom-thiolate (RSAu) model, (b), tilted from the surface normal by $65^{\circ}$, and the (RSAu) polymer, $1 \mathrm{ad}+1 \mathrm{va}$ and $\mathrm{Au}$ adatom-dithiolate (RS-Au-SR) complexes are tilted in the range of $51-57^{\circ}$, which reflects the strong coordination environment of sulfur in the adsorption models.

For electronic structure analysis, we have investigated the partial density of states (PDOS) of the $3 p$ orbitals of the $\mathrm{S}$ 


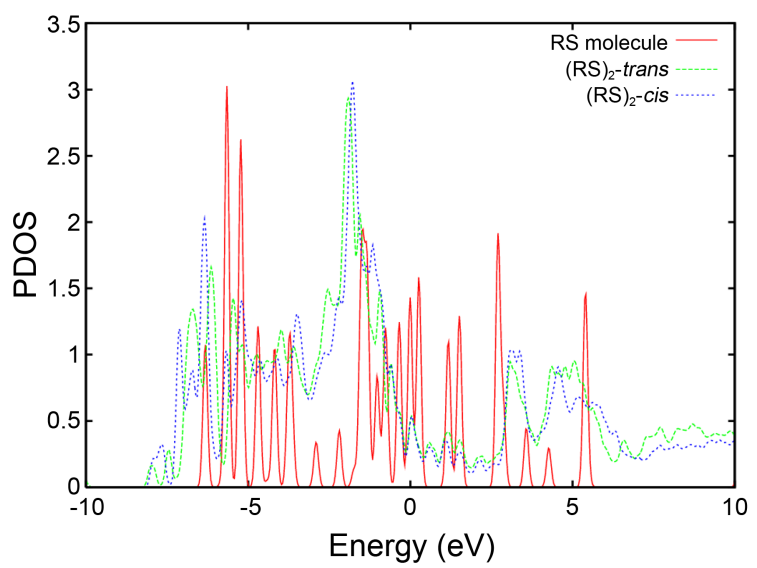

Figure 3. Projected density of states on the $p$ orbitals of the $\mathrm{S}$ atom for the RS, (RS) $)_{2}$-cis, and (RS) $)_{2}$-trans models.

atom, associated with the chemical bonding between the butylthiolate and the $\mathrm{Au}(111)$ surface in the nine structural models. Figure 3 shows the changes in PDOS, which reflect the hybridization of the substrate and the adsorbate orbitals and the degree of bonding responsible for the adsorption. A series of sharp peaks of RS undergo a significant delocalization on the surface, indicative of a strong electronic coupling between the molecule and the surface.

\section{Conclusions}

We have studied the adsorption structures of butylthiolate (ButS) on $\mathrm{Au}(111)$ after introducing adatoms to consider surface reconstruction based on the $c(4 \times 2)$ superlattice. The most stable adsorption structure is the two ButS$\mathrm{Au}$ (adatom)-SBut staple motifs adsorbed in the cisconfiguration $\left((\mathrm{RS})_{2}\right.$-cis model, $\left.(\mathrm{g})\right)$. The $(\mathrm{RS})_{2}$-cis model is $0.17 \mathrm{eV}$ more stable than the unreconstructed surface model in which four ButS molecules adsorbed identically on the fcc-brd site. Due to the steric hindrance between butylthiolate chains, the cis configuration is slightly preferred to the trans configuration for better packing on $\mathrm{Au}(111)$. The present study demonstrates that slab calculations with local basis set can be successfully applied to suggest the expected stability of adatom modified surfaces of $\mathrm{Au}(111)$ for butylthiolate in line with the experimental observation. ${ }^{22}$ It is not clear whether the adatom motifs would become unfavorable or not for a long alkyl chain.

Acknowledgments. This work was supported by grants (2009-0076263, 2010-0001632) from the National Research Foundation of Korea. This work was also supported by a KBSI grant (T3109A) to Y.K.H. Computational resources were provided by the supercomputing center of the Korea Institute of Science and Technology Information (KISTI).

\section{References}

1. Ulman, A. Chem. Rev. 1996, 96, 1533.

2. Schreiber, F. Prog. Surf. Sci. 2000, 65, 151.
3. Love, J. C.; Estroff, L. A.; Kriebel, J. K.; Nuzzo, R. G.; Whitesides, G. M. Chem. Rev. 2005, 105, 1103.

4. Vericat, C.; Vela, M. E.; Salvarezza, R. C. Phys. Chem. Chem. Phys. 2005, 7, 3258.

5. Strong, L.; Whitesides, G. M. Langmuir 1988, 4, 546.

6. Chidsey, C. E. D.; Liu, G. Y.; Rowntree, P.; Scoles, G. J. Chem. Phys. 1989, 91, 4421.

7. Nuzzo, R. G.; Korenic, E. M.; Dubois, L. H. J. Chem. Phys. 1990, 93, 767.

8. Widrig, C. A.; Alves, C. A.; Porter, M. D. J. Am. Chem. Soc. 1991, 113, 2805.

9. Camillone, N.; Chidsey, C. E. D.; Liu, G. Y.; Scoles, G. J. Chem. Phys. 1993, 98, 3503.

10. Fenter, P.; Eisenberger, P.; Liang, K. S. Phys. Rev. Lett. 1993, 70, 2447.

11. Woodruff, D. P. Phys. Chem. Chem. Phys. 2008, 10, 7211.

12. Chaudhuri, A.; Lerotholi, T. J.; Jackson, D. C.; Woodruff, D. P.; Jones, R. G. Phys. Rev. B 2009, 79, 195439.

13. Hayashi, T.; Morikawa, Y.; Nozoye, H. J. Chem. Phys. 2001, 114, 7615.

14. Vargas, M. C.; Giannozzi, P.; Selloni, A.; Scoles, G. J. Phys. Chem. B 2001, 105, 9509.

15. Akinaga, Y.; Nakajima, T.; Hirao, K. J. Chem. Phys. 2001, 114, 8555.

16. Gottschalck, J.; Hammer, B. J. Chem. Phys. 2002, 116, 784.

17. Yourdshahyan, Y.; Rappe, A. M. J. Chem. Phys. 2002, 117, 825.

18. Cao, Y. P.; Ge, Q. F.; Dyer, D. J.; Wang, L. C. J. Phys. Chem. B 2003, 107, 3803 .

19. Kondoh, H.; Iwasaki, M.; Shimada, T.; Amemiya, K.; Yokoyama, T.; Ohta, T.; Shimomura, M.; Kono, S. Phys. Rev. Lett. 2003, 90, 066102.

20. Roper, M. G.; Skegg, M. P.; Fisher, C. J.; Lee, J. J.; Dhanak, V. R.; Woodruff, D. P.; Jones, R. G. Chem. Phys. Lett. 2004, 389, 87.

21. Yu, M.; Bovet, N.; Satterley, C. J.; Bengio, S.; Lovelock, K. R. J.; Milligan, P. K.; Jones, R. G.; Woodruff, D. P.; Dhanak, V. Phys. Rev. Lett. 2006, 97, 166102.

22. Maksymovych, P.; Sorescu, D. C.; Yates, J. T. Phys. Rev. Lett. 2006, $97,146103$.

23. Chaudhuri, A.; Odelius, M.; Jones, R. G.; Lee, T. L.; Detlefs, B.; Woodruff, D. P. J. Chem. Phys. 2009, 130, 124708.

24. Chaudhuri, A.; Lerotholi, T. J.; Jackson, D. C.; Woodruff, D. P.; Dhanak, V. Phys. Rev. Lett. 2009, 102, 126101.

25. Molina, L. M.; Hammer, B. Chem. Phys. Lett. 2002, 360, 264.

26. Grönbeck, H.; Häkkinen, H. J. Phys. Chem. B 2007, 111, 3325.

27. Cossaro, A.; Mazzarello, R.; Rousseau, R.; Casalis, L.; Verdini, A.; Kohlmeyer, A.; Floreano, L.; Scandolo, S.; Morgante, A.; Klein, M. L.; Scoles, G. Science 2008, 321, 943.

28. Wang, J. G.; Selloni, A. J. Phys. Chem. C 2007, 111, 12149.

29. Grönbeck, H.; Häkkinen, H.; Whetten, R. L. J. Phys. Chem. C 2008, 112, 15940 .

30. Artacho, E.; Sanchez-Portal, D.; Ordejon, P.; Garcia, A.; Soler, J. M. Phys. Status Solidi B 1999, 215, 809.

31. Ordejon, P.; Artacho, E. J. M. Soler, Phys. Rev. B 1996, 53, 10441.

32. Perdew, J. P.; Yue, W. Phys. Rev. B 1986, 33, 8800.

33. Perdew, J. P.; Chevary, J. A.; Vosko, S. H.; Jackson, K. A.; Pederson, M. R.; Singh, D. J.; Fiolhais, C. Phys. Rev. B 1992, 46, 6671.

34. Hu, C. D.; Langreth, D. C. Phys. Scr. 1985, 32, 391.

35. Langreth, D. C.; Perdew, J. P. Phys. Rev. B 1980, 21, 5469.

36. Perdew, J. P.; Burke, K.; Ernzerhof, M. Phys. Rev. Lett. 1996, 77, 3865.

37. Troullier, N.; Martins, J. L. Phys. Rev. B 1991, 43, 1993.

38. Franke, A.; Pehlke, E. Phys. Rev. B 2009, 79, 235441.

39. Monkhorst, H. J.; Pack, J. D. Phys. Rev. B 1976, 13, 5188.

40. Grönbeck, H. J. Phys. Chem. C 2010, 114, 15973.

41. Voznyy, O.; Dubowski, J. J. Langmuir 2009, 25, 7353.

42. Jadzinsky, P. D.; Calero, G.; Ackerson, C. J.; Bushnell, D. A.; Kornberg, R. D. Science 2007, 318, 430.

43. Jiang, D. E.; Dai, S. J. Phys. Chem. C 2009, 113, 3763. 